\title{
Effects of stimulus alternations on extinguishing a discrimination
}

\author{
JAMES H. MCCROSKERY, PATRICIA M. OLSEN, and JULIA WALLACE* \\ State University of New York, Oswego, New York 13126
}

\begin{abstract}
Rats were trained in a light ON vs light OFF discrimination in operant chambers with food reinforcement. Following acquisition, extinction under conditions of no alternations between $S+$ and $S-$ and under conditions of numerous alternations between $S+$ and $S$ - were examined. In Experiment I, extinction in $S+$ or $S-$ alone produced less responding to $S+$ and more responding to $S-$ than extinction with regular alternations between $S+$ and $S-$. In Experiment II, 9, 39, or 79 alternations in extinction between $\mathrm{S}+$ and $\mathrm{S}-$ produced no differences in responding. These results indicate that during extinction of a discrimination there is (a) sharpening of differential performance, (b) a difference between multiple- and single-stimulus procedures, and (c) little effect of different numbers of alternations.
\end{abstract}

Extinguishing a discrimination refers to discontinued reinforcement of $\mathrm{S}+$ responding following differential training to St (reinforced stimulus) and $S-$ (nonreinforced stimulus). Procedurally, then, the typical postdiscrimination stimulus generalization test is a form of extinguishing a discrimination, with the added feature that new test stimuli along the training dimension as well as $\mathbf{S}+$ and $\mathbf{S}-$ are presented. Stimulus generalization is tested in extinction so as to exclude the effects of reinforcement and thereby obtain a "purer" assessment of stimulus control; however, the effects of nonreinforcement must also be taken into account. One striking feature of extinguishing a discrimination that has been noted is the increase in differential performance over that of acquisition. While the overall rate of responding to $\mathrm{S}+$ and $\mathrm{S}-$ is declining, the decrease in responding to $\mathrm{S}-$ is relatively greater than to $\mathrm{S}+$, such that the $\mathbf{S}+\mathbf{S}-$ ratio actually increases (Smith \& Hoy, 1954; Gollub \& Urban, 1958; McCroskery, 1972). This same kind of phenomenon has been reported with stimulus generalization testing (Guttman, 1959; Thomas, 1962; Friedman \& Guttman, 1965). In addition to providing further evidence for this enhancement of differential performance, the present experiments were designed to investigate the effects of stimulus alternations on extinguishing a discrimination and relate them to stimulus generalization testing in extinction.

\section{EXPERIMENT I}

It has been shown with pigeons that following intradimensional discrimination training the stimulus control evidenced by the generalization gradient is clearly a function of the test procedure used to assess stimulus control (Donahoe, McCroskery, \& Richardson, 1970). When an alternating quasirandom sequence of stimuli (multiple-stimulus procedure) is presented within the same session to the same $S$, the resulting response

\footnotetext{
*Now at State University of New York, Center at Binghamton.
}

gradient shows the typical shift in the mode of responding beyond St (peak shift) and minimal responding to $S-$ (steepening of the gradient). On the other hand, when one or another stimulus (single-stimulus procedure) is presented for the whole session to different groups of Ss, the result is less responding to $S+$ and enhanced responding to $S_{-}$, giving a more shallow gradient. This multiple-stimulus vs single-stimulus procedure in generalization testing is similar to $\mathbf{S}+$ and $\mathrm{S}-$ alternations (double-stimulus) vs $\mathbf{S}+$ only (single-stimulus) procedures in extinguishing a discrimination (Senf \& Miller, 1967; Ison \& Krane, 1969; Krane \& Ison, 1970, 1971; McCroskery, 1972). Senf and Miller attributed the greater responding in extinction to $\mathrm{S}+$ with the double-stimulus alternating procedure to induction effects (Pavlov, 1927, pp. 188-196). With positive induction, $S+$ responding increases following the presentation of $\mathrm{S}-$ $(\mathrm{S}+|\mathrm{S}->\mathrm{S}+| \mathrm{S}+)$; with negative induction, $\mathrm{S}-$ responding decreases following the presentation of $S+$ $(\mathrm{S}-|\mathrm{S}+<\mathrm{S}-| \mathrm{S}-)$. Based on these considerations, it follows that an alternation in extinction of $\mathrm{S}+$ and $\mathrm{S}-$ (double-stimulus procedure) should produce a steeper gradient than a procedure in which $\mathbf{S}+$ alone is presented to one group of Ss and $S-$ alone to another group (single-stimulus procedure).

\section{Method}

\section{Subjects}

Thirty male Holtzman rats, obtained from Holtzman Company, Madison, Wisconsin, approximately 120 days old, with an average free-feeding weight of $403 \mathrm{~g}$, were used as Ss. The Ss had previously been used in a nondiscrimination runway experiment with sucrose reinforcement. Following termination of that study, they were given full access to food for 1 week before being reduced to $85 \%$ of the 120 -day free-feeding weight, at which they were maintained for the duration of the experiment.

\section{Apparatus}

Three identical operant chambers (Lehigh Valley Electronics. LVE 143-03) were used. The test chambers were enclosed in separate sound-dampening cubicles with blowers for ventilation. 


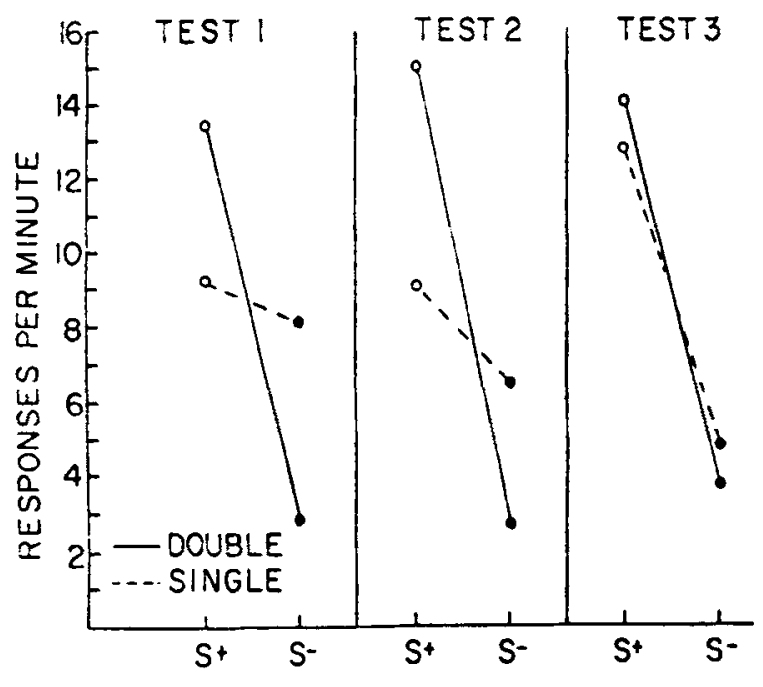

Fig. 1. Comparisons of response rates to $S+$ and $S$ - between the double- and single-stimulus procedures on the three extinction test sessions.

A houselight affixed to the ceiling of each cubicle was on continuously. The discriminative stimulus was a $1 / 2$-in.-diam frosted white light located above the response lever. Light $O N$ was $S+$ and light OFF was $S$ - for all Ss. Reinforcing stimuli were 45-mg Noyes food pellets. Experimental events were controlled by .standard relay and timing equipment, and responses were monitored by impulse counters.

\section{Procedure}

There were four sessions of pretraining ( 30 food pellets per session) in the presence of $S+: 1$ day of feeder training, 1 day of barpress training, 1 day of continuous reinforcement, and 1 day of variable-interval 20-sec reinforced responding. Discrimination training consisted of 201 -min alternating periods between $\mathrm{S}+$ and $\mathrm{S}$-. During the $10 \mathrm{St}$ periods, lever responses were reinforced on a variable-interval 20-sec schedule, and during the $10 \mathrm{~S}$ - periods, no lever responses were reinforced. Ten sessions of discrimination training preceded Extinction Test 1. Two additional sessions of discrimination training preceded Extinction Test 2, which was followed by two more sessions of discrimination training prior to Extinction Test 3. Each extinction session was directly preceded by $4 \mathrm{~min}$ of regular $\mathrm{S}+$ and $S$ - periods, with reinforcement in $S+$. During the subsequent $20 \mathrm{~min}$, no responses were reinforced.

For each extinction test, the animals were assigned (independent of responding) to one of three conditions $(N=10)$. There were two single-stimulus groups: (1) St only, in which the $S+$ stimulus was presented for the full $20 \mathrm{~min}$, and (2) $\mathrm{S}-$ only, in which the $S$ - stimulus was presented for the full $20 \mathrm{~min}$. The double-stimulus group received 101 -min $S+$ presentations alternating with $101-\min S$ - presentations. In both acquisition and extinction, the animals were run in squads of three. For extinction the conditions were assigned such that each condition was represented in each squad. Conditions per chamber were balanced across squads. In order to make within- as well as between-S comparisons, no $S$ had the same extinction condition twice. Therefore, of the 10 animals that received double-stimulus extinction in the second test, five had received single-stimulus $\mathrm{S}+$ in the first test and five had received single-stimulus $S$ - in the first test. On the third test, each animal was assigned to the extinction condition that he had not yet received.

\section{Results}

During acquisition a session record was kept of the number of responses to $S+$ and $S$ - for each $S$. During the three extinction tests, a minute by minute record was kept of responding to whatever stimulus was present during that minute. In making comparisons between the double-stimulus (D-S) and single-stimulus (S-S) conditions, only the 10-min (every other) periods for the single-stimulus groups corresponding to the appropriate minutes for the double-stimulus group were evaluated.

\section{Session Total}

By the tenth session of discrimination training, the animals were making an average of 24 responses per minute to $S+$ and an average of 10 responses per minute to $\mathbf{S}-$. In proportional terms (S+/total), they were responding at a .70 level of differential performance. The gradients for the first extinction test (Session 11) are depicted in the left panel of Fig. 1. Differential performance in extinction for single $S s$ can be determined from the double-stimulus condition: There was an increase to .82 . Because the D-S group had S+ and $S-$ measures from the same $S s$ whereas the single-stimulus measures represent separate groups, a series of $t$ tests was made for each extinction test on the four critical comparisons. As anticipated, D-S St responding was greater than $S \cdot S$ St responding $[t(18)=3.09, p<.01]$ and D-S $S-$ responding was less than S-S $S-$ responding $[t(18)=2.62, \quad p<.02]$. Responding to $S+$ and $S-$ did not differ for the two single-stimulus groups $(\mathrm{t}<1)$, whereas there is clear differential responding in the D-S group $[t(9)=8.51$, $\mathrm{p}<.001]$.

Following two more sessions of discrimination training, the Ss were averaging 28.7 responses per minute to $S+$ and 12.4 responses per minute to $S-$, giving a .70 proportion (Session 13). On the following day of extinction, proportional responding increased to .85 . As depicted in the middle panel of Fig. 1, the results of the second extinction test are basically the same as in Test 1: D-S St responding was greater than S-S S+ responding $[\mathrm{t}(18)=2.88, \mathrm{p}<.01]$, D-S $\mathrm{S}-$ responding was less than $S-S S-$ responding $[t(18)=3.48, p<.01]$, S-S S+ and S-S S- did not differ $[\mathrm{t}(18)=1.47, \mathrm{p}>.10]$, and there was differential responding in the D-S group $[t(9)=9.51, p<.001]$.

After the final two sessions of discrimination training, the animals were responding at higher levels. S+ rate reached 33.0 , while $S-$ rate was 12.7 , giving a .73 proportion (Session 16). Again, in the following extinction test, the D-S Ss showed an increase to .80 . By the third extinction test, however, the differences noted in the two previous tests had disappeared: As seen in the right panel of Fig. 1, D-S and S-S did not differ in responding to $S+(t<1)$ or in responding to $S-$ $[\mathrm{t}(18)=1.45, \mathrm{p}>.10]$. By the third test session there appears to be single-stimulus control of responding, for the S-S S+ group responded significantly more than the S-S S- group $[\mathrm{t}(18)=3.29, \mathrm{p}<.01]$, just as within the 
Table 1

Mean Number of Responses by Comparison Minutes to $S+$ and $S-$ for the Double-Stimulus (D-S) and the Single-Stimulus (S-S) Groups on the Three Extinction Tests*

\begin{tabular}{|c|c|c|c|c|c|c|c|c|c|c|c|c|}
\hline & & \multicolumn{10}{|c|}{ Minutes } & \multirow[b]{2}{*}{ ANOVA } \\
\hline & & 1 & 2 & 3 & 4 & 5 & 6 & 7 & 8 & 9 & 10 & \\
\hline Test 1 & $\begin{array}{l}\text { S-S S+ } \\
\text { D-S S+ } \\
\text { D-S S- } \\
\text { S-S S- }\end{array}$ & $\begin{array}{r}9.1 \\
11.9\end{array}$ & $\begin{array}{r}17.0 \\
21.2 \\
5.2 \\
9.1\end{array}$ & $\begin{array}{r}9.6 \\
17.7 \\
4.4 \\
11.8\end{array}$ & $\begin{array}{r}9.8 \\
15.9 \\
2.7 \\
11.6\end{array}$ & $\begin{array}{r}7.9 \\
11.8 \\
1.2 \\
5.1\end{array}$ & $\begin{array}{l}7.4 \\
9.7 \\
2.3 \\
8.6\end{array}$ & $\begin{array}{l}5.1 \\
8.4 \\
1.4 \\
5.2\end{array}$ & $\begin{array}{l}5.8 \\
7.4 \\
1.2 \\
7.4\end{array}$ & $\begin{array}{l}4.0 \\
5.4 \\
1.7 \\
5.6\end{array}$ & $\begin{array}{r}3.0 \\
7.7 \\
.6 \\
4.6\end{array}$ & $\begin{array}{l}\mathrm{S}_{+}=9.98, \mathrm{p}<.01 \\
\mathrm{~S}_{-}=14.45, \mathrm{p}<.01\end{array}$ \\
\hline Test 2 & $\begin{array}{l}\text { S-S S+ } \\
\text { D-S S+ } \\
\text { D-S S- } \\
\text { S-S S- }\end{array}$ & $\begin{array}{r}7.2 \\
15.6\end{array}$ & $\begin{array}{r}17.3 \\
27.7 \\
4.6 \\
10.3\end{array}$ & $\begin{array}{r}12.1 \\
18.0 \\
1.7 \\
7.4\end{array}$ & $\begin{array}{r}9.2 \\
16.6 \\
4.1 \\
6.7\end{array}$ & $\begin{array}{r}5.2 \\
18.4 \\
2.3 \\
5.5\end{array}$ & $\begin{array}{l}5.5 \\
7.0 \\
1.5 \\
5.5\end{array}$ & $\begin{array}{l}4.8 \\
5.1 \\
3.0 \\
4.2\end{array}$ & $\begin{array}{r}2.8 \\
10.5 \\
.6 \\
3.5\end{array}$ & $\begin{array}{r}4.4 \\
7.2 \\
.5 \\
4.3\end{array}$ & $\begin{array}{l}2.5 \\
6.5 \\
1.5 \\
1.1\end{array}$ & $\begin{array}{l}S+=8.72, p<.01 \\
S-=16.05, p<.001\end{array}$ \\
\hline \multirow[b]{2}{*}{ Test 3} & $\begin{array}{l}\text { S-S S+ } \\
\text { D-S S+ }\end{array}$ & & $\begin{array}{l}27.1 \\
23.3\end{array}$ & $\begin{array}{l}21.4 \\
23.4\end{array}$ & $\begin{array}{l}11.2 \\
18.1\end{array}$ & $\begin{array}{l}8.9 \\
9.8\end{array}$ & $\begin{array}{l}7.0 \\
8.6\end{array}$ & $\begin{array}{l}4.7 \\
9.0\end{array}$ & $\begin{array}{l}3.1 \\
5.9\end{array}$ & $\begin{array}{l}3.1 \\
4.3\end{array}$ & $\begin{array}{l}3.2 \\
7.6\end{array}$ & $.78, p>.20$ \\
\hline & $\begin{array}{l}\text { D-S S- } \\
\text { S-S S- }\end{array}$ & $\begin{array}{l}10.1 \\
15.1\end{array}$ & $\begin{array}{r}6.1 \\
10.3\end{array}$ & $\begin{array}{l}6.5 \\
8.4\end{array}$ & $\begin{array}{l}5.9 \\
5.9\end{array}$ & $\begin{array}{l}1.8 \\
3.3\end{array}$ & $\begin{array}{l}1.5 \\
2.0\end{array}$ & $\begin{array}{l}2.0 \\
2.2\end{array}$ & $\begin{array}{r}.2 \\
1.4\end{array}$ & $\begin{array}{r}1.4 \\
.7\end{array}$ & $\begin{array}{l}2.4 \\
2.1\end{array}$ & $S_{-}=1.69, \mathrm{p}>.20$ \\
\hline
\end{tabular}

*On the right-hand side are the between-group $F$ ratios $(d f=1 / 18)$ of comparisons between D-S and $S$-S to $S+$ and $S-$ in each test.

D.S group responding was clearly differential $[\mathrm{t}(9)=7.39, \mathrm{p}>.001]$.

Since each $S$ received extinction under all three conditions, within-S comparisons were also made on responding to St and S- under D.S and S-S. The between-S comparisons are supported by the within-S results: Ss responded more to $S+$ in D-S than in S-S $[t(29)=3.52, p<.001]$ and less to $S-$ in D.S than in S-S $[\mathrm{t}(29)=5.59, \mathrm{p}<.001$.

\section{Minute by Minute}

Since a minute by minute record was kept of responding in each extinction session, the overall D.S vs S-S comparisons can be examined in more detail. The minute by minute means for both comparisons on all three tests are presented in Table 1. Since the first minute of extinction for both the S-S S+ group and the D-S group involved a transition from the previous $S$ - of the warm-up period, this minute was excluded from the analysis. In all three extinction tests, there was no difference between D-S and S-S in responding to St on this first minute of extinction (all ps $>.10$ ). The minute by minute data were evaluated by analysis of variance ( 2 by 9 for the $S+$ comparisons and 2 by 10 for the $S-$ comparisons). The six between-group comparisons are presented in the appropriate rows of Table 1. As can be seen, these results are consistent with the $t$ comparisons already presented. In all six comparisons, there was a decrease in responding over minutes (all ps $<.001$ ). On Extinction Tests 1 and 2, where the D-S and S-S groups differed, these differences were constant since there was no Groups by Minutes interaction (all ps $>.10$ ).

\section{Discussion}

These results are further indication that the procedures used to assess differential responding in extinction may produce different rates of responding to
St and $S-$. In all three extinction tests, differential performance was greater than that of the previous acquisition session. As predicted from relating comparisons of stimulus alternations in discrimination extinction (Senf \& Miller, 1967; Ison \& Krane, 1969; Krane \& Ison, 1970, 1971) to postdiscrimination generalization testing (Donahoe, McCroskery, \& Richardson, 1970), with double-stimulus extinction there was more responding to $\mathrm{S}+$ and less responding to $\mathrm{S}$ - than with single-stimulus extinction. However, in the present experiment these differences disappeared over the three testing sessions. From the present experiment, it cannot be determined whether additional testing or extended training produced Test 3 single-stimulus results more like double-stimulus results. There is evidence (Terrace, 1966) that acquisition contrast effects and extinction generalization effects disappear after extended training with pigeons. However, Krane and Ison (1970) found greater S+ responding in extinction with the double-stimulus procedure only after extended training and concluded that extinction "induction" is different from acquisition contrast. Nonetheless, the present results suggest that with rats these alternation vs nonalternation differences may be limited to low and middle levels of S+ responding.

One explanation of these differences in extinction between double- and single-stimulus procedures is that alternations in the double-stimulus procedure induce an $\mathrm{S}+$ increase (positive induction) and an $\mathrm{S}-$ decrease (negative induction). This application of induction (Pavlov, 1927) has been invoked for discrimination extinction by Senf and Miller (1967). On the other hand, Krane and Ison $(1970,1971)$ have argued that "positive induction" results are due to $\mathrm{S}$ - presentation in the alternating, double-stimulus condition preventing the build-up of inhibition across successive $S+$ trials, as occurs in the nonalternating, $\mathbf{S}+$ only condition. One purpose of the second experiment was to attempt to analyze the effects of various numbers of stimulus alternations in discrimination extinction. 


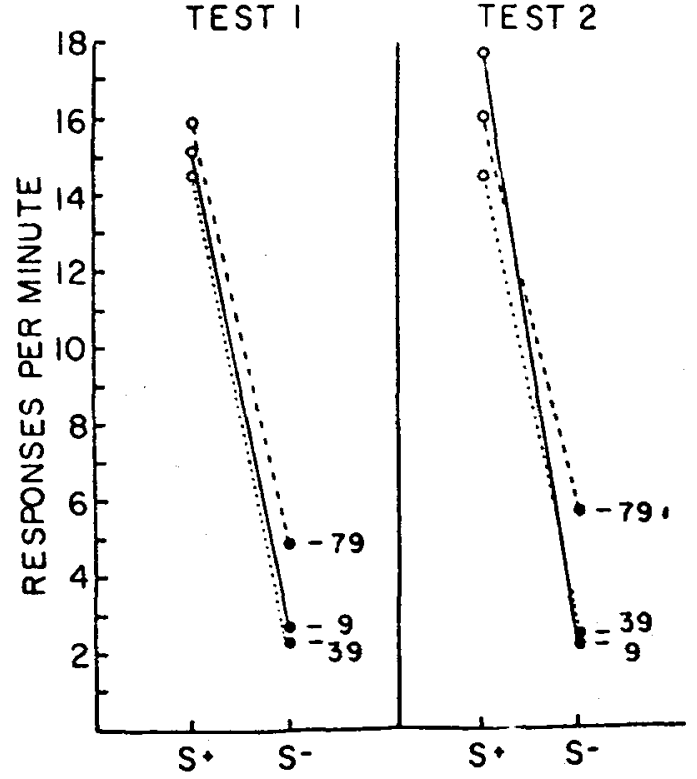

Fig 2. Comparisons of response rates to $S+$ and $S$ - between groups receiving 9,39 , or 79 stimulus alternations on the two extinction test sessions.

\section{EXPERIMENT II}

If in Experiment I S+ responding was augmented by the alternation from $S-$ and $S-$ responding was diminished by the alternation from $S+$ in the double-stimulus group, then perhaps the slope of the gradient would vary as a function of the number of alternations between $S+$ and $S-$-, with many alternations producing a steeper gradient. That is, if the alternation from $\mathrm{S}-$ to $\mathrm{S}+$ produces a temporary increase in responding to $S+$, then $S s$ given many alternations to $S+$ and $S$ - should total more responses then $S s$ given few alternations from $\mathrm{S}-$ to $\mathrm{S}+$. Likewise, if the transition from $S+$ to $S-$ induces a temporary decrease in responding to $S-$, then numerous alternations from $S+$ to $S-$ should produce fewer total responses to $S-$ in $S s$ given many alternations than in Ss given few alternations.

\section{Method}

\section{Subjects and Apparatus}

Twenty-seven male Holtzman rats obtained from Holtzman Company, Madison, Wisconsin, approximately 140 days old, with an average free-feeding weight of $402 \mathrm{~g}$, were used as Ss. The rats had previously been used in an E-maze pilot study with food-pellet reinforcement. Following termination of that study, they were given full access to food for 1 week prior to being reduced to $85 \%$ of the 140 -day free-feeding weight, at which they were maintained for the duration of the experiment. The apparatus used in Experiment II was identical to that used in Experiment I.

\section{Procedure}

Pretraining and discrimination training were identical to that of Experiment I. Each extinction session was directly preceded by $4 \mathrm{~min}$ of regular $\mathrm{S}+$ and $\mathrm{S}$ - alternating periods with reinforcement in S+. For extinction testing, the Ss were assigned (independent of responding) to one of three conditions $(\mathrm{N}=9)$ : Group 9 received five $2-\min S+$ periods alternating with five 2-min $\mathrm{S}$ - periods, giving nine transitions in extinction; Group 39 was given $2030-\mathrm{sec} S+$ periods alternating with 2030 -sec Speriods, giving 39 alternations; and Group 79 received 4015 -sec $\mathrm{S}+$ periods alternating with $4015-\sec S-$ periods, giving 79 transitions. As such, all groups were presented with $10 \mathrm{~min}$ of $\mathrm{S}+$ and $10 \mathrm{~min}$ of $\mathrm{S}-$ in extinction. The first extinction test was given after 10 sessions of discrimination training. The animals were run in squads of three; all three animals in a squad were assigned to the same extinction group. Following the first extinction test, there were five additional discrimination training sessions. before the second extinction test. On the second test, all Ss received the same treatment conditions as they had received on the first test.

\section{Results}

By the tenth session of discrimination training, the animals were making an average of 33 responses per minute to $S+$ and 17 responses per minute to $S-$, giving a proportion of .66 . The gradients for the first extinction test (Session 11) are depicted in the left panel of Fig. 2. The S+ average for all three groups was 15.3 and the $S$ - average was 4.1 ; extinction test proportional responding increased to .80 . These averages were given because an analysis of variance ( 3 by 2 ) showed no difference between groups $(F<1)$, a difference in responding to stimuli $[F(1,24)=130.79, p<.001]$, and no Groups by Stimuli interaction $(F<1)$.

After the next five sessions of discrimination training, the animals were making an average of 40 responses per minute to $S+$ and 14 responses per minute to $S-$, giving a proportion of .77 . The gradients for the second extinction test (Session 17) are depicted in the right panel of Fig. 2. The S+ average for all three groups was 16.2 and the $S-$ average was 3.6; extinction test proportional responding increased to .84 . Again, these averages were given because an analysis of variance ( 3 by 2 ) showed no difference between groups $(F<1)$, a difference in responding to stimuli $[F(1,24)=114.50$, $\mathrm{p}<.001]$, and no Groups by Stimuli interaction $[F(2,24)=1.76, p>.10]$.

\section{Discussion}

The results of the second experiment strongly indicate that the number of stimulus alternations has little effect upon the rate of responding to $S+$ and $S-$ in extinction at the acquisition levels attained and within the alternation range tested. This finding for discrimination extinction is consistent with stimulus generalization testing results; investigators frequently give many short exposures to the test stimuli, thereby giving more alternations in extinction than during acquisition training (e.g., Hanson, 1959). There was no evidence for the systematic differences predicted as a function of number of alternations. One caution, however, should be placed on comparisons between Experiments I and II. By the first test session (Session 11), the Ss in the 
second experiment were responding at a higher level to St than the Ss in the first experiment (33 vs 24 responses per minute). Since it was suggested that single-stimulus vs double-stimulus differences in rats may disappear with high $\mathrm{S}+$ rates, Experiment II may not have been an adequate test for the predicted alternation-induced induction effects. The possibility of the number of alternations affecting the steepness of the gradient should be tested over a more complete range of acquisition levels.

Despite these cautions, the disappearance of a singlevs double-stimulus difference by the third test of Experiment I along with the lack of difference of alternations in Experiment II suggests that the focus should not be on alternation induction effects as an explanation for single- vs double-stimulus differences. Donahoe, McCroskery, and Richardson (1970) found that induction effects (e.g., $S-|S+<S-| S-)$ contributed only a small portion to the large multiple- vs single-stimulus differences that were obtained. Krane and Ison (1971) conclude that it is the performance of the nonalternation single-stimulus group that should be explained. The focus should not only be on those conditions that increase double-stimulus control in extinction but also on those conditions that impair single-stimulus control in extinction. As such, the terms "comparison" (for multiple-stimulus procedures) and "noncomparison" (for single-stimulus procedures) used by Hiss and Thomas (1963) seem very suitable. Stimulus change per se in extinction may be more critical than the number of stimulus changes. It may be that the primary function of stimulus alternations in extinction is to cue differential responding rather than to induce rate increments or decrements.

Together, the two studies indicate the relationship between generalization testing in extinction and extinguishing a discrimination. The sharpening of differential performance during extinction was obtained, the difference between double- and single-stimulus extinction procedures was demonstrated, and the lack of effect of differences in multiple alternations was shown. It would seem fruitful to further study extinction of a discrimination before analyzing the more complex effects of additional stimuli as in multiple-stimulus generalization testing.

\section{REFERENCES}

Donahoe, J. W., McCroskery, J. H., \& Richardson, W. K. Effects of context on the postdiscrimination gradient of stimulus generalization. Journal of Experimental Psychology, 1970, 84, 58-63.

Friedman, H., \& Guttman, N. Further analysis of discrimination training on stimuius generalization gradients. In D. I. Mostofsky (Ed.), Stimulus generalization. Stanford: Stanford University Press, 1965. Pp. 255-267.

Gollub, L. R., \& Urban, J. T. The accentuation of a rate difference during extinction. Journal of the Experimental Analysis of Behavior, 1958, 1, 365-369.

Guttman, H. Generalization gradients around stimuli associated with different schedules of reinforcement. Journal of Experimental Psychology, 1959, 58, 343-350.

Hanson, H. Effects of discrimination training on stimulus generalization. Journal of Experimental Psychology, 1959, 58 , 321-334.

Hiss, R. H., \& Thomas, D. R. Stimulus generalization as a function of testing procedure and response measures. Journal of Experimental Psychology, 1963, 65, 587-592.

Ison, J. R., \& Krane, R. V. Induction in differential instrumental conditioning. Journal of Experimental Psychology, 1969, 80, 183-185.

Krane, R. V., \& Ison, J. R. Positive induction in differential instrumental conditioning: Effects of extended acquisition training. Journal of Comparative \& Physiological Psychology, $1970,73,334-340$

Krane, R. V., \& Ison, J. R. Positive induction in differential instrumental conditioning: Effects of the interstimulus interval. Journal of Comparative \& Physiological Psychology, $1971,75,129-135$.

McCroskery, J. H. Extinguishing, abolishing, and reversing discriminative performance. Journal of General Psychology, 1972, 86, 25-37.

Pavlov, I. P. Conditioned reflexes (Anrep trans.). London: Oxford University Press, 1927.

Senf, G. M., \& Miller, N. E. Evidence for positive induction in discrimination learning. Journal of Comparative \& Physiological Psychology, 1967, 64, 121-127.

Smith, M. H., \& Hoy, W. J. Rates of responding during operant discrimination. Journal of Experimental Psychology, 1954. $48,259-264$

Terrace, H. S. Behavior contrast and the peak shift: Effects of extended discrimination training. Journal of the Experimental Analysis of Behavior, 1966, 9, 613-617.

Thomas, D. R. The effects of drive and discrimination training on stimulus generalization. Journal of Experimental Psychology, 1962, 64, 24-28.

(Received for publication May 26, 1973; revision accepted August 15, 1973.) 